\title{
Combinatorial expansions in $K$-theoretic bases
}

\author{
Jason Bandlow \\ Google, Inc. \\ San Bruno, California, U.S.A. \\ jbandlow@gmail.com
}

\author{
Jennifer Morse * \\ Department of Mathematics \\ Drexel University \\ Philadelphia, PA, U.S.A. \\ morsej@math.drexel .edu
}

Submitted: May 6, 2012; Accepted: Dec 5, 2012; Published: Dec 13, 2012

Mathematics Subject Classifications: 05A19; 05E05; 19E20

\begin{abstract}
We study the class $\mathcal{C}$ of symmetric functions whose coefficients in the Schur basis can be described by generating functions for sets of tableaux with fixed shape. Included in this class are the Hall-Littlewood polynomials, $k$-Schur functions, and Stanley symmetric functions; functions whose Schur coefficients encode combinatorial, representation theoretic and geometric information. While Schur functions represent the cohomology of the Grassmannian variety of $G L_{n}$, Grothendieck functions $\left\{G_{\lambda}\right\}$ represent the $K$-theory of the same space. In this paper, we give a combinatorial description of the coefficients when any element of $\mathcal{C}$ is expanded in the $G$-basis or the basis dual to $\left\{G_{\lambda}\right\}$.
\end{abstract}

\section{Introduction}

Schubert calculus uses intersection theory to convert enumerative problems in projective geometry into computations in cohomology rings. In turn, the representation of Schubert classes by Schur polynomials enables such computations to be carried out explicitly. The combinatorial theory of Schur functions is central in the application of Schubert calculus to problems in geometry, representation theory, and physics.

In a similar spirit, a family of power series called Grothendieck polynomials were introduced by Lascoux and Schützenberger in [23] to explicitly access the $K$-theory of $G L_{n} / B$. In [6], Fomin and Kirillov first studied the stable limit of Grothendieck polynomials as $n \rightarrow \infty$. When indexed by Grassmannian elements, we call these limits the $G$-functions. Grothendieck polynomials and $G$-functions are connected to representation theory and geometry in a way that leads to a generalization of Schubert calculus where combinatorics is again at the forefront. Moreover, fundamental aspects of the theory of Schur

${ }^{*}$ Research supported in part by NSF grants DMS:1001898,0652641,0638625 
functions are contained in the theory of $G$-functions since each $G_{\lambda}$ is an inhomogeneous symmetric polynomial whose lowest homogeneous component is the Schur function $s_{\lambda}$.

Parallel to the study of $G$-functions is the study of a second family of functions that arise by duality with respect to the Hall inner product on the ring $\Lambda$ of symmetric functions. In particular, results in $[13,3]$ imply that the $G$-functions form a Schauder basis for the completion of $\Lambda$ with respect to the filtration by the ideals $\Lambda^{r}=\bigoplus_{|\lambda| \geqslant r} \mathbb{Z} s_{\lambda}$. The dual Hopf algebra to this completion is isomorphic to $\Lambda$. Therein lies the basis of $g$-functions, defined by their duality to the $G$-basis. Lam and Pylyavskyy first studied these functions directly in [18] where they were called dual stable Grothendieck polynomials. By duality, each $g_{\lambda}$ is inhomogeneous with highest homogeneous component equal to $s_{\lambda}$.

Strictly speaking, the $G$ - and $g$-functions do not lie in the same space and there is no sensible way to write $G$-functions in terms of $g$-functions. However, any element of $\Lambda$ can be expanded into both the $G$ - and the $g$-functions and it is such expansions that are of interest here. Motivated by the many families of symmetric functions whose transition matrices with Schur functions have combinatorial descriptions and encode representation theoretic or geometric information, our focus is on functions with what we refer to as tableaux Schur expansions. A symmetric function, $f_{\alpha}$, is said to have a tableaux Schur expansion if there is a set of tableaux $\mathbb{T}(\alpha)$ and a weight function $w t_{\alpha}$ so that

$$
f_{\alpha}=\sum_{T \in \mathbb{T}(\alpha)} w t_{\alpha}(T) s_{s h(T)}
$$

Among the classical examples is the family of Hall-Littlewood polynomials [10, 14], whose tableaux Schur expansion gives the decomposition of a graded character of $S L_{n}(\mathbb{C})$ into its irreducible components [8]. A more recent example is given by the $k$-atoms [15]. These are conjectured to represent Schubert classes for the homology of the affine Grassmannian when $t=1$ and their very definition is a tableaux Schur expansion.

In this paper, we give combinatorial descriptions for the $G$ - and the $g$-expansion of every function with a tableaux Schur expansion. Our formulas are in terms of set-valued tableaux and reverse plane partitions; $G$-functions are the weight generating functions of the former and $g$-functions are the weight generating functions of the latter. More precisely, for any given set $\mathbb{T}(\alpha)$ of semistandard tableaux, we describe associated sets $\mathbb{S}(\alpha)$ and $\mathbb{R}(\alpha)$ of set-valued tableaux and reverse plane partitions, respectively. Given also any function $w t_{\alpha}$ on $\mathbb{T}(\alpha)$, we define an extension of $w t_{\alpha}$ to $\mathbb{S}(\alpha)$ and $\mathbb{R}(\alpha)$. In these terms, we prove that any function $f_{\alpha}$ satisfying (1) can be expanded as

$$
f_{\alpha}=\sum_{S \in \mathbb{S}(\alpha)} w t_{\alpha}(S)(-1)^{\varepsilon(S)} g_{s h(S)}=\sum_{R \in \mathbb{R}(\alpha)} w t_{\alpha}(R) G_{s h(R)} .
$$

The construction of sets $\mathbb{S}(\alpha)$ and $\mathbb{R}(\alpha)$ is described in section 3 and the proof of $(2)$ is given in section 4 .

Since a Schur function has a trivial tableaux Schur expansion, the simplest application of (2) describes the transition matrices between $G / g$ and Schur functions in terms of certain reverse plane partitions and set-valued tableaux. These transition matrices 
were alternatively described by Lenart in [13] using certain skew semistandard tableaux. Although our description is not obviously equinumerous, we give a bijective proof of the equivalence in section 5. As a by-product, we show that Lenart's theorem follows from (2).

In section 6 , we show how the description of a $G / g$-expansion given by (2) may lead to a more direct combinatorial interpretation for the expansion coefficients. For example, we show that the Hall-Littlewood functions can be defined by extending the notion of charge to reverse plane partitions and set-valued tableaux. We also show that the $G / g$ expansions of a product of Schur functions can be described by certain Yamanouchi reverse plane partitions and set-valued tableaux. Note, this is not the $G$-expansion of a product of $G$-functions which was settled in [3].

We use (2) to get the $G$ and $g$-expansions for $k$-atoms and Stanley symmetric functions in section 7 and leave as open problems their further simplification. We finish with a curious identity which has a simple proof using the methods described here.

\section{Definitions and notation}

\subsection{Symmetric function basics}

We begin by setting our notation and giving standard definitions (see eg. [25, 30, 2] for complete details on symmetric functions).

Definition 2.1. The Ferrers diagram of a partition $\lambda=\left(\lambda_{1}, \lambda_{2}, \cdots, \lambda_{k}\right)$ is a left- and bottom-justified array of $1 \times 1$ square cells in the first quadrant of the coordinate plane, with $\lambda_{i}$ cells in the $i^{\text {th }}$ row from the bottom.

Example 2.2. The Ferrers diagram of the partition $(3,2)$ is

Given any partition $\lambda$, the conjugate $\lambda^{\prime}$ is the partition obtained by reflecting the diagram of $\lambda$ about the line $y=x$. For example, the conjugate of $(3,2)$ is $(2,2,1)$.

Definition 2.3. A semistandard tableau of shape $\lambda$ is a filling of the cells in the Ferrers diagram of $\lambda$ with positive integers, such that the entries

- are weakly increasing while moving rightward across any row, and

- are strictly increasing while moving up any column.

Example 2.4. A semistandard tableau of shape $(3,2)$ is $\frac{23}{111^{2}}$.

Throughout this paper, the letter $T$ will generally refer to a tableau, and $\mathbb{T}$ will typically denote a set of tableaux.

The evaluation of a semistandard tableau is the sequence $\left(\alpha_{i}\right)_{i \in \mathbb{N}}$ where $\alpha_{i}$ is the number of cells containing $i$. The evaluation of the tableau in Example 2.4 is $(2,2,1)$ (it is customary to omit trailing 0 's). We use $S S T(\lambda)$ to denote the set of all semistandard tableaux of shape $\lambda$, and $S S T(\lambda, \mu)$ to denote the set of all semistandard tableaux of shape $\lambda$ and evaluation $\mu$. 
Definition 2.5. A word is a finite sequence of positive integers. The reading word of a tableau $T$, which we denote by $w(T)$, is the sequence $\left(w_{1}, w_{2}, \ldots, w_{n}\right)$ obtained by listing the elements of $T$ starting from the top-left corner, reading across each row, and then continuing down the rows.

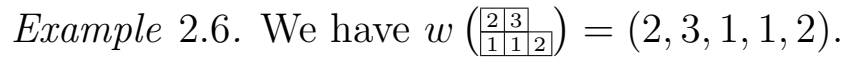

We use the fundamental operations jeu-de-taquin [28] and $R S K$-insertion [4, 27, 11] on words. The reader can find complete details and definitions of these operations in $[21,30,7]$. A key property of $R S K$-insertion is that

$$
\operatorname{RSK}(w(T))=T,
$$

for any tableau $T$. When two words insert to the same tableau under the RSK map, they are said to be Knuth equivalent.

The weight generating function of semistandard tableaux can be used as the definition of Schur functions. For any tableau $T$, let $\mathbf{x}^{e v(T)}=x_{1}^{\alpha_{1}} x_{2}^{\alpha_{2}} \cdots$, where $\left(\alpha_{1}, \alpha_{2}, \cdots\right)$ is the evaluation of $T$.

Definition 2.7. The Schur function $s_{\lambda}$ is defined by

$$
s_{\lambda}=\sum_{T \in S S T(\lambda)} \mathbf{x}^{e v(T)} .
$$

The Schur functions are elements of $\mathbb{Z}\left[\left[x_{1}, x_{2}, \cdots\right]\right]$, the power series ring in infinitely many variables, and are well known to be a basis for the symmetric functions (i.e., those elements of $\mathbb{Z}\left[\left[x_{1}, x_{2}, \cdots\right]\right]$ which are invariant under any permutation of their indices).

Example 2.8. The Schur function $s_{(2,1)}$ is

$$
s_{(2,1)}=x_{1}^{2} x_{2}+x_{1} x_{2}^{2}+2 x_{1} x_{2} x_{3}+\cdots
$$

corresponding to the tableaux

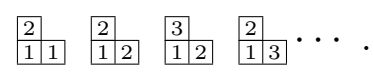

Another basis for the symmetric functions is given by the monomial symmetric functions.

Definition 2.9. The monomial symmetric function $m_{\lambda}$ is defined by

$$
m_{\lambda}=\sum_{\alpha} \mathbf{x}^{\alpha}
$$

summing over all distinct sequences $\alpha$ which are a rearrangement of the parts of $\lambda$. (Here $\lambda$ is thought of as having finitely many non-zero parts, followed by infinitely many 0 parts.) 
Example 2.10. The monomial symmetric function $m_{(2,1)}$ is

$$
m_{(2,1)}=x_{1}^{2} x_{2}+x_{1} x_{2}^{2}+x_{1}^{2} x_{3}+x_{1} x_{3}^{2}+x_{2}^{2} x_{3}+x_{2} x_{3}^{2}+\ldots
$$

The Kostka numbers give the change of basis matrix between the Schur and monomial symmetric functions. For two partitions $\lambda$, $\mu$, we define the number $K_{\lambda, \mu}$ to be the number of semistandard tableaux of shape $\lambda$ and weight $\mu$. From the previous definitions, one can see that a consequence of the symmetry of the Schur functions is that

$$
s_{\lambda}=\sum_{\mu} K_{\lambda, \mu} m_{\mu}
$$

There is a standard inner product on the vector space of symmetric functions (known as the Hall inner product), defined by setting

$$
\left\langle s_{\lambda}, s_{\mu}\right\rangle=\left\{\begin{array}{lc}
1 & \text { if } \lambda=\mu \\
0 & \text { otherwise }
\end{array}\right.
$$

The following proposition is a basic, but very useful, fact of linear algebra.

Proposition 2.11. If $\left(\left\{a_{\lambda}\right\},\left\{a_{\lambda}^{*}\right\}\right)$ and $\left(\left\{b_{\lambda}\right\},\left\{b_{\lambda}^{*}\right\}\right)$ are two pairs of dual bases for an inner-product space, and

$$
a_{\lambda}=\sum_{\mu} M_{\lambda, \mu} b_{\mu}
$$

then

$$
b_{\mu}^{*}=\sum_{\lambda} M_{\lambda, \mu} a_{\lambda}^{*}
$$

Proof. Pairing both sides of (4) with $b_{\mu}^{*}$ gives $\left\langle a_{\lambda}, b_{\mu}^{*}\right\rangle=M_{\lambda, \mu}$. Similarly, pairing both sides of (5) with $a_{\lambda}$ gives $\left\langle b_{\mu}^{*}, a_{\lambda}\right\rangle=M_{\lambda, \mu}$. (4) and (5) are thus equivalent.

The set of complete homogeneous symmetric functions, $\left\{h_{\lambda}\right\}$, are defined to be the basis that is dual to the monomial symmetric functions. An immediate consequence of (3) and Proposition 2.11 is that

$$
h_{\mu}=\sum_{\lambda} K_{\lambda, \mu} s_{\lambda}
$$

\section{$2.2 \quad G$-functions}

Buch introduced the combinatorial notion of set-valued tableaux in [3] to give a new characterization for $G$-functions and to prove an explicit formula for the structure constants of the Grothendieck ring of a Grassmannian variety with respect to its basis of Schubert structure sheaves. It is this definition of $G$-functions we use here. 
Definition 2.12. A set-valued tableau of shape $\lambda$ is a filling of the cells in the Ferrers diagram of $\lambda$ with sets of positive integers, such that

- the maximum element in any cell is weakly smaller than the minimum element of the cell to its right, and

- the maximum element in any cell is strictly smaller than the minimum entry of the cell above it.

Another way to view this definition is by saying that the selection of a single element from each cell (in any possible way) will always give a semistandard tableau.

Example 2.13. A set-valued tableau of shape $(3,2)$ is

$$
S=\begin{array}{|r|r|r|}
\hline 3 & 4,5,6 & \\
\hline 1,2 & 2,3 & 3 \\
\hline
\end{array}
$$

We have omitted the set braces, ' $\{$ ' and ' $\}$ ', here and throughout for clarity of exposition.

The evaluation of a set-valued tableau $S$ is the composition $\alpha=\left(\alpha_{i}\right)_{i \geqslant 1}$ where $\alpha_{i}$ is the total number of times $i$ appears in $S$. The evaluation of the tableau in Example 2.13 is $(1,2,3,1,1,1)$. The collection of all set-valued tableaux of shape $\lambda$ will be denoted $S V T(\lambda)$, and the subset of these with evaluation $\alpha$ will be denoted $S V T(\lambda, \alpha)$. We write $k_{\lambda, \mu}$ for the number of set-valued tableaux of shape $\lambda$ and evaluation $\mu$. We will typically denote a set-valued tableau with the letter $S$. Finally, we define the sign of a set-valued tableau, $\varepsilon(S)$, to be the number elements minus the number of cells:

$$
\varepsilon(S)=|\operatorname{ev}(S)|-|\operatorname{shape}(S)| .
$$

A multicell will refer to a cell in $S$ that contains more than one letter. Note that when $S$ has no multicells we view $S$, as a usual semistandard tableau. In this case, $|e v(S)|=|\operatorname{shape}(S)|$, and $\varepsilon(S)=0$.

Definition 2.14. For any partition $\lambda$, the Grothendieck function $G_{\lambda}$ is defined by

$$
G_{\lambda}=\sum_{\mu}(-1)^{|\mu|-|\lambda|} k_{\lambda, \mu} m_{\mu}=\sum_{S \in S V T(\lambda)}(-1)^{\varepsilon(S)} x^{e v(S)}
$$

For terms where $|\mu|=|\lambda|, k_{\lambda \mu}=K_{\lambda \mu}$ since there are no multicells. Hence $G_{\lambda}$ equals $s_{\lambda}$ plus higher degree terms. Since the $G_{\lambda}$ are known to be symmetric functions, they therefore form a basis for the appropriate completion of $\Lambda$.

Applying Proposition 2.11 to this definition gives rise to the basis $\left\{g_{\lambda}\right\}$ that is dual to $\left\{G_{\lambda}\right\}$ by way of the system,

$$
h_{\mu}=\sum_{\mu}(-1)^{|\mu|-|\lambda|} k_{\lambda, \mu} g_{\lambda}
$$

over partitions $\mu$. These $g$-functions $g_{\lambda}$ were studied explicitly by Lam and Pylyavskyy in [18] where they showed that $g_{\lambda}$ can be described as a certain weight generating function for reverse plane partitions. 
Definition 2.15. A reverse plane partition of shape $\lambda$ is a filling of the cells in the Ferrers diagram of $\lambda$ with positive integers, such that the entries are weakly increasing in rows and columns.

Example 2.16. A reverse plane partition of shape $(3,2)$ is $\frac{12}{\frac{1}{112} 2}$.

Following Lam and Pylyavskyy (and differing from some other conventions) we define the evaluation of a reverse plane partition to be the composition $\alpha=\left(\alpha_{i}\right)_{i \geqslant 1}$ where $\alpha_{i}$ is the total number of columns in which $i$ appears. The evaluation of the reverse plane partition in Example 2.16 is $(2,2)$. The collection of all reverse plane partitions of shape $\lambda$ will be denoted $R P P(\lambda)$ and the subset of these with evaluation $\alpha$ will be denoted $R P P(\lambda, \alpha)$. We will typically use the letter $R$ to refer to a reverse plane partition.

Theorem 2.17 (Lam-Pylyavskyy). The polynomials $g_{\lambda}$ have the expansion

$$
g_{\lambda}=\sum_{R \in R P P(\lambda)} \mathbf{x}^{e v(R)} .
$$

We note that when $|\mu|=|\lambda|$, the entries must be strictly increasing up columns; hence $g_{\lambda}$ is equal to $s_{\lambda}$ plus lower degree terms.

\section{General formula for $K$-theoretic expansions}

Our combinatorial formula for the $G$ - and the $g$-expansion of any function with a tableaux Schur expansion is in terms of reverse plane partitions and set-valued tableaux, respectively. The formula relies on a natural association of these objects with semistandard tableaux which comes about by a careful choice of reading word for set-valued tableaux and reverse plane partitions.

Definition 3.1. The reading word of a set-valued tableau $S$, denoted by $w(S)$, is the sequence $\left(w_{1}, w_{2}, \ldots, w_{n}\right)$ obtained by listing the elements of $S$ starting from the top-left corner, reading each row according to the following procedure, and then continuing down the rows. In each row, we first ignore the smallest element of each cell, and read the remaining elements from right to left and from largest to smallest within each cell. Then we read the smallest element of each cell from left to right, and proceed to the next row.

Example 3.2. The reading word of the set-valued tableau $S$ in Example 2.13 is $w(S)=$ $(6,5,3,4,3,2,1,2,3)$.

Definition 3.3. Given a reverse plane partition $R$, circle in each column only the bottommost occurrence of each letter. The reading word of $R$, which we denote by $w(R)$, is the sequence $\left(w_{1}, w_{2}, \ldots, w_{n}\right)$ obtained by listing the circled elements of $R$ starting from the top-left corner, and reading across each row and then continuing down the rows.

Example 3.4. The reverse plane partition $R$ in Example 2.16 has $w(R)=(2,1,1,2)$.

THE ELECTRONiC JOURNAL OF COMBINATORICS 19(4) (2012), \#P39 
This given, for a set $\mathbb{T}(\alpha)$ of semistandard tableaux, we define sets $\mathbb{S}(\alpha)$ and $\mathbb{R}(\alpha)$ of set-valued tableaux and reverse plane partitions, respectively, by

$$
\begin{gathered}
S \in \mathbb{S}(\alpha) \text { if and only if } R S K(w(S)) \in \mathbb{T}(\alpha), \text { and } \\
R \in \mathbb{R}(\alpha) \text { if and only if } R S K(w(R)) \in \mathbb{T}(\alpha) .
\end{gathered}
$$

Similarly, we can extend any function $w t_{\alpha}$ defined on $\mathbb{T}(\alpha)$ to $\mathbb{S}(\alpha)$ and $\mathbb{R}(\alpha)$ by setting

$$
w t_{\alpha}(X)=w t_{\alpha}(R S K(w(X)))
$$

for any $X$ in $\mathbb{S}(\alpha)$ or $\mathbb{R}(\alpha)$.

It is in terms of these definitions that we express the $G$ - and $g$-expansions for functions with a tableaux-Schur expansion.

Theorem 3.5. Given $f_{\alpha}$ with a tableaux Schur expansion,

$$
f_{\alpha}=\sum_{T \in \mathbb{T}(\alpha)} w t_{\alpha}(T) s_{s h(T)}
$$

we have

$$
\begin{aligned}
f_{\alpha} & =\sum_{R \in \mathbb{R}(\alpha)} w t_{\alpha}(R) G_{s h(R)} \\
& =\sum_{S \in \mathbb{S}(\alpha)} w t_{\alpha}(S)(-1)^{\varepsilon(S)} g_{s h(S)} .
\end{aligned}
$$

Proof. Writing the $s_{s h(T)}$ on the right hand side of equation (8) as the sum over tableaux gives

$$
f_{\alpha}=\sum_{T \in \mathbb{T}(\alpha)} w t_{\alpha}(T) \sum_{T^{\prime} \in S S T(\operatorname{sh}(T))} \mathbf{x}^{e v\left(T^{\prime}\right)} .
$$

Similarly, writing the $g_{s h(S)}$ in (10) as the sum over reverse plane partitions as given by Theorem 2.17, we obtain

$$
\sum_{S \in \mathbb{S}(\alpha)} w t_{\alpha}(S)(-1)^{\varepsilon(S)} g_{s h(S)}=\sum_{S \in \mathbb{S}(\alpha)} w t_{\alpha}(S)(-1)^{\varepsilon(S)} \sum_{R \in R P P(\operatorname{sh}(S))} \mathbf{x}^{e v(R)} .
$$

Since every semistandard tableau can be viewed as a set-valued tableau and as a reverse plane partition, every monomial term in (11) also appears as a term in (12). Thus to prove that (11) equals (12), it suffices to show that the terms in (12) not occurring in (11) sum to zero.

In the same way, writing the $G_{s h(S)}$ in $(9)$ as the sum over set-valued tableaux according to Definition 2.14, we find that

$$
\sum_{R \in \mathbb{R}(\alpha)} w t_{\alpha}(R) G_{s h(R)}=\sum_{R \in \mathbb{R}(\alpha)} w t_{\alpha}(R) \sum_{S \in S V T(\operatorname{sh}(R))}(-1)^{\varepsilon(S)} \mathbf{x}^{e v(S)}
$$


Again, every term in (11) appears in (13) and it suffices to show that the extra terms in (13) sum to zero.

From these observations, we can simultaneously prove that (11) equals (12) and (13) by producing a single sign-reversing and weight-preserving involution. To be precise, in the next section we introduce a map $\iota(S, R)=\left(S^{\prime}, R^{\prime}\right)$ and prove that it is an involution on the set of pairs of $(S, R)$, where $S$ is a set-valued tableau and $R$ is a reverse plane partition of the same shape, satisfying the properties:

1. $\iota(S, R)=(S, R)$ if and only if $S$ and $R$ are both semistandard tableaux,

2. $\varepsilon(S)=\varepsilon\left(S^{\prime}\right) \pm 1$ when $S$ is not a semistandard tableau, and

3. $(R S K(w(S)), R S K(w(R)))=\left(R S K\left(w\left(S^{\prime}\right)\right), R S K\left(w\left(R^{\prime}\right)\right)\right)$.

\section{The involution}

We introduce basic operations on set-valued tableaux and reverse plane partitions called dilation and contraction and will then define the involution $\iota$ in these terms. To this end, first setting some notation for set-valued tableaux and reverse plane partitions will be helpful.

Given a set-valued tableau $S$, let $\operatorname{row}(S)$ be the highest row containing a multicell. Let $S_{>i}$ denote the subtableau formed by taking only rows of $S$ lying strictly higher than row $i$. For a reverse plane partition $R$, let $\operatorname{row}(R)$ denote the highest row containing an entry that lies directly below an equal entry. We use the convention that when $S$ has no multicell, $\operatorname{row}(S)=0$ and when no column of $R$ has a repeated entry, $\operatorname{row}(R)=0$.

Definition 4.1. Given a set-valued tableau $S$, let $c$ be the rightmost multicell in $\operatorname{row}(S)$ and define $x=x(S)$ to be the largest entry in $c$. The dilation of $S, \operatorname{di}(S)$, is constructed from $S$ by removing $x$ from $c$ and inserting it, via $R S K$, into $S_{>\operatorname{row}(S)}$.

Example 4.2. Since $\operatorname{row}(S)=2$ and $x(S)=6$,

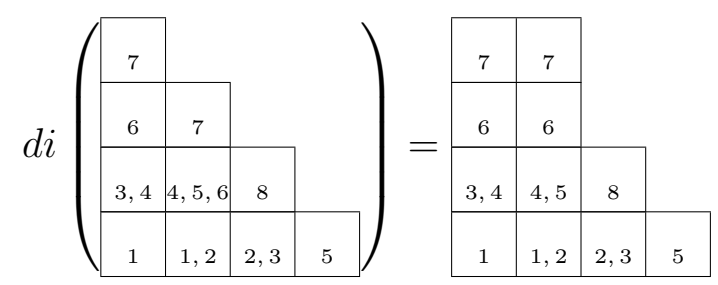

Property 4.3. For any set-valued tableau $S$, di $(S)$ is a set-valued tableau.

Proof. Let $c$ be the rightmost multicell in row $i$ and let $x=x(S)$. Rows weakly lower than row $i$ in $S^{\prime}=d i(S)$ form a set-valued tableau since $S$ is set-valued to start. For rows above row $i$, first note that the cell above $c$ is empty or contains a letter strictly greater than $x$. Thus, the insertion of $x$ into row $i+1$ puts $x$ in a cell that is weakly to the left of $c$. Moreover, all entries in row $i$ of $S^{\prime}$ that are weakly to the left of cell $c$ are strictly smaller than $x$ since $c$ is a multicell in $S$. Thus, in $S^{\prime}, x$ is strictly larger than all entries in the cell below it. The claim then follows from usual properties of RSK insertion. 
Property 4.4. For any set-valued tableaux $S$ and $S^{\prime}=\operatorname{di}(S)$,

$$
R S K(w(S))=R S K\left(w\left(S^{\prime}\right)\right) .
$$

Proof. Let $i=\operatorname{row}(S)$. For some word $v, w(S)$ can be factored as $w\left(S_{>i}\right) \cdot x(S) \cdot v$ since $x(S)$ is the first letter in the reading word of row $i$. The definition of dilation then gives that the word of $S^{\prime}$ is $w\left(S_{>i} \leftarrow x\right) \cdot v$. Thus the Knuth equivalence classes of $w(S)$ and $w\left(S^{\prime}\right)$ are the same since RSK insertion preserves Knuth equivalence.

We remark that it is Property 4.4 which motivated our definition for the reading word of a set-valued tableau.

Definition 4.5. Given a reverse plane partition $R$, let $i=\operatorname{row}(R)$ and let $c$ be the rightmost cell in row $i+1$ that contains the same entry as the cell below it. The contraction of $R, c o(R)$, is constructed by replacing $c$ with a marker and using reverse jeu-de-taquin to slide this marker up and to the right until it exits the diagram.

Example 4.6.

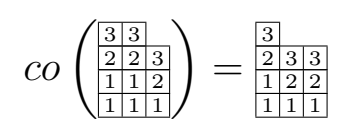

Property 4.7. For any reverse plane partitions $R$ and $R^{\prime}=c o(R)$,

$$
R S K(w(R))=R S K\left(w\left(R^{\prime}\right)\right) .
$$

Proof. Let $i=\operatorname{row}(R)$ and note that the portion of the reading word of $R$ obtained by reading rows weakly below row $i$ is unchanged by contraction. Moreover, the rows of $R$ higher than $i+1$ form a semistandard tableau and thus the jeu-de-taquin moves in these rows preserve Knuth equivalence. In row $i+1$, any initial rightward slide of the marker does not change the reading word since every letter to the right of the marker is strictly greater than the letter below it (and hence, strictly greater than the letter below it and to its left). It thus suffices to check that the move taking the marker from row $i+1$ to row $i+2$ preserves Knuth equivalence. To this end, let $\widehat{R}$ denote the stage of the jeu-de-taquin process at which the next move takes the empty marker from row $i+1$ to $i+2$. Consider the subtableau $\widehat{R}^{*}$ consisting only of the letters in rows $i+1$ and $i+2$ which contribute to the reading word. In general, the form of $\widehat{R}^{*}$ will be

$$
\begin{array}{|l|l|l|}
\hline u & x & v \\
\hline w & \bullet & y \\
\hline
\end{array}
$$

where $y, v$ are weakly increasing words with $\ell(y) \geqslant \ell(v)$ and each $y_{i}<v_{i}, x$ is a letter with $x \leqslant y_{1}<v_{1}$, and $u, w$ are weakly increasing words with $\ell(w) \leqslant \ell(u)$ (since an entry of $w$ may not be the lowest in its column and thus not part of the reading word whereas all entries of $u$ contribute to reading word since they are in row $i+2)$. It remains to show the Knuth equivalence of the words uxvwy and uvwxy. We first note that the insertion of the word $u w$ is

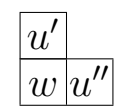

THE ELECTRONic Journal of COMBinatorics 19(4) (2012), \#P39 
for some decomposition of $u$ into disjoint subwords $u^{\prime}, u^{\prime \prime}$. With this observation, it is not hard to verify that both words uxvwy and uvwxy insert to

$$
\begin{array}{|l|l|l|l|}
\hline u^{\prime} & v & & \\
\hline w & u^{\prime \prime} & x & y \\
\hline
\end{array}
$$

and hence these words are Knuth equivalent.

Definition 4.8. For a set-valued tableau $S$ and a reverse plane partition $R$ of the same shape as $S$, define the map

$$
\iota:(S, R) \rightarrow\left(S^{\prime}, R^{\prime}\right)
$$

according to the following four cases (where $y(S, R)$, and the dilation and contraction of a pair are defined below):

1. if $\operatorname{row}(S)=\operatorname{row}(R)=0$, the pair $(S, R)$ is a fixed point

2. if $i=\operatorname{row}(S)>\operatorname{row}(R)$, the pair is dilated

3. if $i=\operatorname{row}(R)>\operatorname{row}(S)$, the pair is contracted

4. if $\operatorname{row}(R)=\operatorname{row}(S)$, the pair is dilated when $x(S) \geqslant y(S, R)$ and is otherwise contracted.

In case (2), $S^{\prime}=\operatorname{di}(S)$ and $R^{\prime}$ is constructed from $R$ by replacing the cell of $R$ in position $(i, j)=S^{\prime} \backslash S$ with an empty marker and sliding this marker to the south-west using jeude-taquin. When the marker reaches row $i$, we replace it by the entry in the cell directly above it. In case (3), $R^{\prime}=c o(R)$. Construct $S^{\prime}$ from $S$ by deleting the cell of $S$ in position $(i, j)=R \backslash R^{\prime}$ and reverse RSK bumping its entry until the entry $y=y(S, R)$ is bumped from row $i+1$. Finally, add entry $y$ to the unique cell of row $i$ where $y$ is maximal in its cell and the non-decreasing row condition is maintained. Case (4) reduces to case (2) or (3), determined by comparing the entry $y(S, R)$ to the number $x(S)$ described in the definition of dilation.

Example 4.9. The involution $\iota$ exchanges the two pairs below:

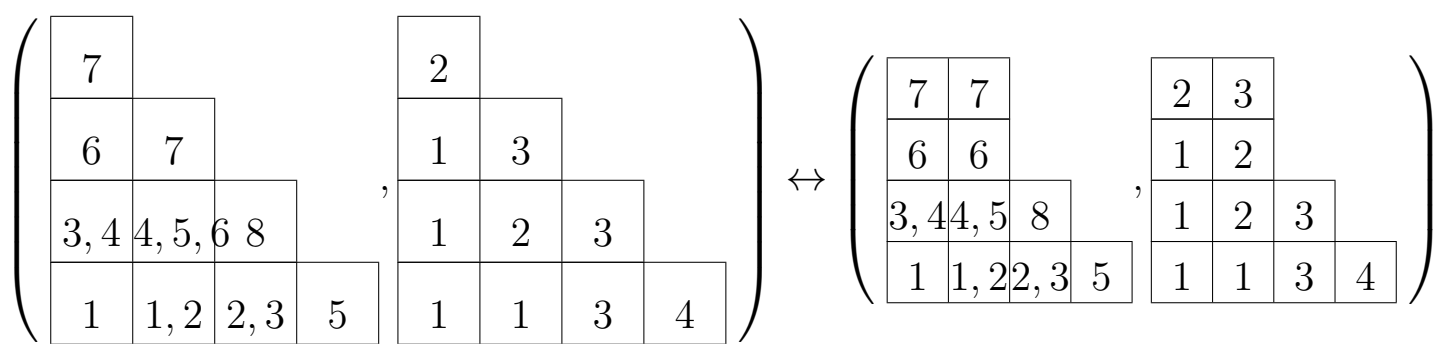

The pair $(S, R)$ on the left has $\operatorname{row}(S)=\operatorname{row}(R)=2$ and $x=y=6$, implying that $(S, R)$ is dilated under $\iota$. The pair $\left(S^{\prime}, R^{\prime}\right)$ on the right has $\operatorname{row}(S)=\operatorname{row}(R)=2$ and $x=5<y=6$ and is thus contracted. Note that $\varepsilon(S)=\varepsilon\left(S^{\prime}\right)+1$ and that

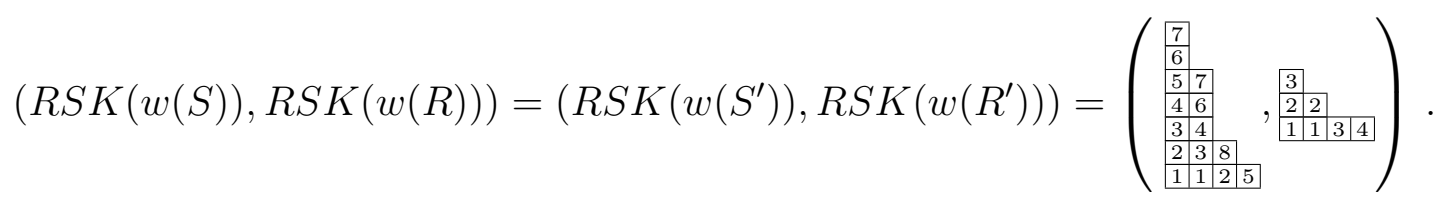


Thus, $\iota$ reverses the sign and preserves the weight of this pair.

Proposition 4.10. The map $\iota$ is a sign-reversing and weight-preserving involution on the set of pairs of $(S, R)$, where $S$ is a set-valued tableau and $R$ is a reverse plane partition of the same shape. The fixed points of $\iota$ are pairs $(S, R)$ where $S$ and $R$ are both semistandard tableau.

Proof. We first verify that $\left(S^{\prime}, R^{\prime}\right)=\iota(S, R)$ is in fact a pair where $S^{\prime}$ is a set-valued tableau and $R^{\prime}$ is a reverse plane partition. When $\iota$ requires dilation, Property 4.3 assures that $S^{\prime}$ is a valid set-valued tableaux and it is straightforward to verify that $R^{\prime}$ is a valid reverse plane partition by properties of jeu-de-taquin.

In the case that $\iota$ involves contraction, $R^{\prime}$ is a reverse plane partition again by properties of jeu-de-taquin. Since $i=\operatorname{row}(R) \geqslant \operatorname{row}(S), S_{>i}$ is a semistandard tableau implying by RSK that $S_{>i}^{\prime}$ is as well and that $y$ is well-defined. It remains to check that there is a unique cell in row $i$ of $S$ into which $y$ can be placed so that it is maximal in this cell and row $i$ maintains the non-decreasing condition. We claim that this cell is the rightmost cell $m$ of row $i$ whose entries are all strictly less than $y$. Note that $m$ exists since the cell of $S$ directly below the cell from which $y$ was bumped has only entries smaller than $y$. We claim that $m$ is the unique cell into which $y$ can be placed; namely, no cell to the right of $m$ contains an element strictly less than $y$. This is clear when $i>\operatorname{row}(S)$ since then $S$ has no multicells in row $i$. Otherwise, conditions of case (4) imply that the largest element of a multicell in row $i$ is $x(S)<y$. Hence there are no multicells to the right of $m$ and the claim follows. Finally, we observe that the entry in the cell directly above $m$ (if it exists) must be strictly greater than $y$, since this cell is weakly to the right of the cell from which $y$ was bumped.

We now show that $\iota$ is indeed an involution by proving that if $\left(S^{\prime}, R^{\prime}\right)$ is obtained by dilation then $\iota\left(S^{\prime}, R^{\prime}\right)$ will require contraction, and vice versa. Consider the case that $\iota$ requires dilation. The definition of row implies that $\operatorname{row}\left(R^{\prime}\right)=i \geqslant \operatorname{row}\left(S^{\prime}\right)$ given $i=\operatorname{row}(S) \geqslant \operatorname{row}(R)$. Further, the reversibility of the RSK algorithm and jeu-de-taquin on semistandard tableaux give that $y\left(R^{\prime}, S^{\prime}\right)=x(S)>x\left(S^{\prime}\right)$. Therefore, applying $\iota$ to $\iota(S, R)$ requires the contraction case. The cases in which $\iota$ requires contraction to start follow similarly.

That $\iota$ is sign-reversing and has the appropriate fixed point set is easy to verify from the definition and it remains only to show that $\iota$ is weight-preserving. From the definition of $\iota$, and the fact that $\iota$ is an involution, we have either:

1. $R^{\prime}=c o(R)$ and $S=\operatorname{di}\left(S^{\prime}\right)$, or

2. $R=\operatorname{co}\left(R^{\prime}\right)$ and $S^{\prime}=\operatorname{di}(S)$.

In either case, that $\iota$ is weight-preserving follows from Properties 4.4 and 4.7.

\section{Schur expansions and an alternate proof}

Lenart proved in [13] that the transition matrices between $G$ and Schur functions have a beautiful combinatorial interpretation in terms of objects that have since been called 
elegant fillings in [18]. Here we show how the simplest application of Theorem 3.5 gives rise to a new interpretation for these transition matrices in terms of certain reverse plane partitions and set-valued tableaux.

Alternatively, we give a bijection between the elegant fillings and these reverse plane partitions/set-valued tableaux. As a by-product, we have an alternate proof for Lenart's result following from Theorem 3.5 and vice versa.

\subsection{A new approach to Schur and $G / g$-transitions}

Definition 5.1. An elegant filling is a skew semistandard tableaux with the property that the numbers in row $i$ are no larger than $i-1$. An elegant filling whose entries are strictly increasing across rows is called strict. We let $f_{\lambda}^{\mu}$ denote the number of elegant fillings of shape $\lambda / \mu$ and $F_{\mu}^{\lambda}$ denote the number of strict elegant fillings of shape $\mu / \lambda$.

Theorem 5.2. [13] The transition matrices between the Schur functions and the Gfunctions are given by the following:

$$
\begin{aligned}
s_{\mu} & =\sum_{\lambda} f_{\lambda}^{\mu} G_{\lambda} \\
G_{\lambda} & =\sum_{\mu}(-1)^{|\lambda|+|\mu|} F_{\mu}^{\lambda} s_{\mu} .
\end{aligned}
$$

Note that the transition between $g$ and Schur functions follows immediately by duality:

$$
\begin{aligned}
& g_{\lambda}=\sum_{\mu} f_{\lambda}^{\mu} s_{\mu} \\
& s_{\mu}=\sum_{\lambda}(-1)^{|\lambda|+|\mu|} F_{\mu}^{\lambda} g_{\lambda} .
\end{aligned}
$$

The simplest application of Theorem 3.5 provides a new combinatorial description for the $f_{\mu}^{\lambda}$ and $F_{\lambda}^{\mu}$ coefficients.

Proposition 5.3. Fix a partition $\lambda$ and a semistandard tableau $T$ of shape $\mu . F_{\mu}^{\lambda}$ is the number of set-valued tableaux of shape $\lambda$ whose reading word is equivalent to $w(T)$ and $f_{\lambda}^{\mu}$ is the number of reverse plane partitions of shape $\lambda$ whose reading word is equivalent to $w(T)$.

Proof. Consider the simple case that $\mathbb{T}$ consists of just one tableau $T$ of shape $\mu$. We can then apply Theorem 3.5 to the trivial expansion

$$
s_{\mu}=\sum_{T \in \mathbb{T}} s_{s h(T)}
$$

to find that

$$
s_{\mu}=\sum_{S \in \mathbb{S}}(-1)^{\varepsilon(S)} g_{s h(S)}=\sum_{R \in \mathbb{R}} G_{s h(R)},
$$


where $\mathbb{S}$ is the set of all set-valued tableaux whose reading word is Knuth equivalent to $w(T)$ and $\mathbb{R}$ is the set of all reverse plane partitions whose reading word is Knuth equivalent to $w(T)$. The result on $F_{\mu}^{\lambda}$ then follows by (17) and the interpretation for $f_{\lambda}^{\mu}$ follows from (14).

\section{$5.2 \quad$ Bijections}

Here we describe bijections between elegant fillings and certain reverse plane partitions, and between strict elegant fillings and certain set-valued tableaux. The bijections lead to alternate proofs for Proposition 5.3, Theorem 5.2, and Theorem 3.5.

We first consider a map on strict elegant fillings. Recall that $\operatorname{row}(S)$ is the highest row of $S$ with a multicell.

Definition 5.4. For any fixed tableau $T$, we define the map

$$
\phi_{T}:\{S \in S V T(\mu): w(S) \sim w(T)\} \rightarrow\{\text { strict elegant fillings of shape } \operatorname{sh}(T) / \mu\}
$$

where $\phi_{T}(S)$ is the filling of $s h(T) / \mu$ that records the sequence of set-valued tableaux

$$
S=S_{0} \rightarrow \operatorname{di}(S)=S_{1} \rightarrow \operatorname{di}\left(S_{1}\right)=S_{2} \rightarrow \cdots \rightarrow S_{r}=T
$$

by putting in cell $\operatorname{sh}\left(S_{i}\right) / \operatorname{sh}\left(S_{i-1}\right)$, the difference between the row index of this cell and $\operatorname{row}\left(S_{i-1}\right)$.

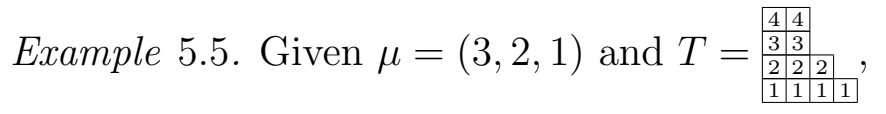

$$
\phi_{T}\left(\begin{array}{|c|c|c}
\hline 4 & \multicolumn{3}{|c}{} \\
\cline { 1 - 2 } 2 & 23 & \\
\hline 1 & 1 & 1234
\end{array}\right)=\begin{array}{|l|l|l|}
\hline 2 & 3 & \\
\hline & 2 & \\
\hline & & 1 \\
\hline & & \\
\hline
\end{array}
$$

is constructed by recording the sequence of dilations

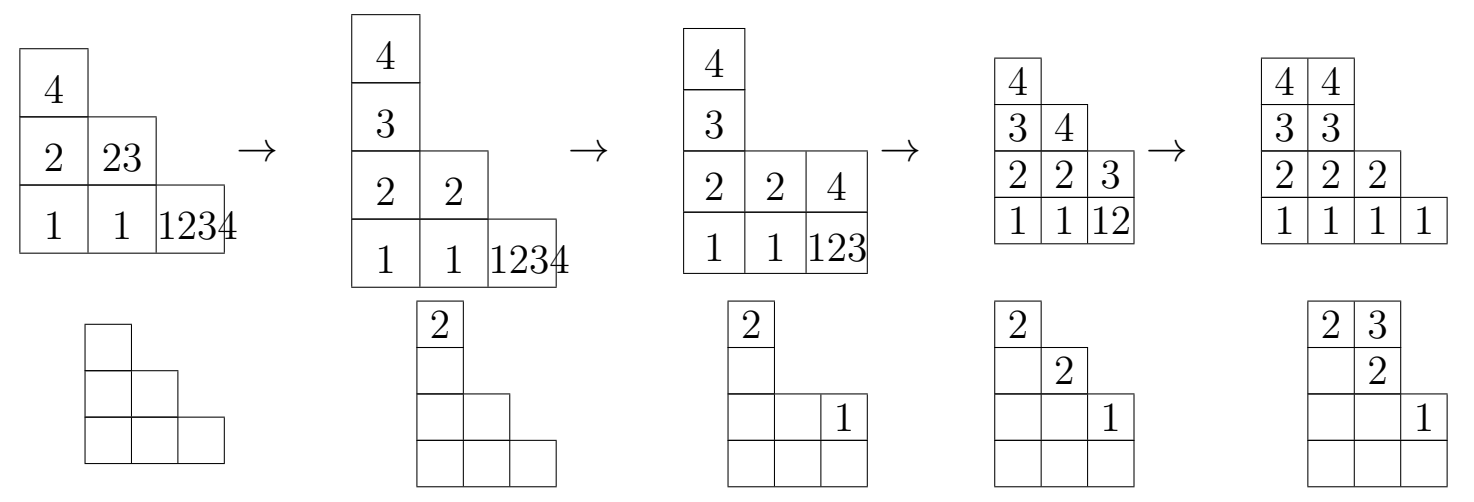


Proposition 5.6. For any tableau $T, \phi_{T}$ is a bijection.

Proof. Fix a tableau $T$ and let $\lambda$ denote its shape. Consider a set-valued tableau $S$ of shape $\mu$ whose reading word is equivalent to $w(T)$.

We start by showing that $F=\phi_{T}(S)$ is a strict elegant filling of shape $\lambda / \mu$. Let

$$
S=S_{0} \rightarrow \operatorname{di}(S)=S_{1} \rightarrow \operatorname{di}\left(S_{1}\right)=S_{2} \rightarrow \cdots \rightarrow S_{r}=T .
$$

Note that this procedure indeed ends with $T$ since each dilation preserves the Knuth equivalence class of the reading word. Since each $\operatorname{sh}\left(S_{i}\right) \subset \operatorname{sh}\left(S_{i+1}\right)$, and $\operatorname{row}\left(S_{i}\right) \geqslant$ $\operatorname{row}\left(S_{i+1}\right), F$ is an elegant filling of the correct shape by construction. To ensure that $F$ is a strict elegant filling, it is enough to know that the bumping paths created by successive dilations starting in the same row do not terminate in the same row. Since dilation starts with the largest entry in a row, such successive dilations involve bumping from row $r$, a letter $z$ after an $x$ where $z \leqslant x$. Therefore, the bumping path created by $x$ must be weakly inside the bumping path of $z$ and in particular, terminates in a higher row.

It remains to show that $\phi_{T}$ is invertible. For the inverse map, consider a strict elegant filling of shape $\lambda / \mu$. In the elegant filling, we first replace each entry $i$ by $r-i$ where $r$ is the row index of the cell containing $i$. The resulting filling consists of "destination rows" for the corresponding entries in our fixed tableau $T$. We proceed by performing contraction on the entries of $T$ which are outside of the inner shape $\mu$, stopping the reverse-bumping procedure when we get to the destination row. The order in which these contractions are performed is determined first by the destination rows (smallest to largest) and then by the height of the original cell (highest to lowest). This concludes the proof.

Recall that $\operatorname{row}(R)$ is the row index of the highest cell in a reverse plane partition $R$ which contains the same entry as the cell immediately above it.

Definition 5.7. For any fixed tableau $T$, we define the map

$$
\psi_{T}:\{R \in R P P(\lambda): w(R) \sim w(T)\} \rightarrow\{\text { elegant fillings of shape } \lambda / \operatorname{sh}(T)\}
$$

where $\psi_{T}(R)$ is the filling of $\lambda / \operatorname{sh}(T)$ that records the sequence of reverse plane partitions

$$
R=R_{0} \rightarrow \operatorname{co}(R)=R_{1} \rightarrow \operatorname{co}\left(R_{1}\right)=R_{2} \rightarrow \cdots \rightarrow R_{r}=T
$$

by putting row $\left(R_{i}\right)$ in cell $\operatorname{sh}\left(R_{i}\right) / \operatorname{sh}\left(R_{i+1}\right)$.

Example 5.8.

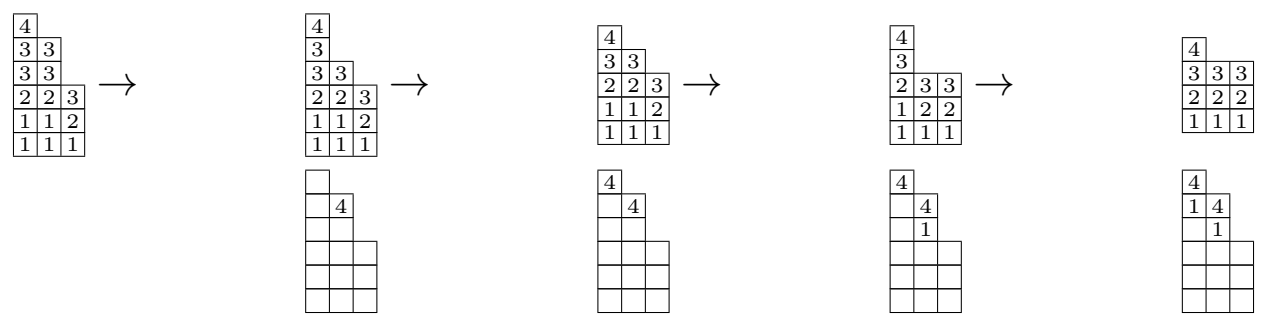


Proposition 5.9. For any semistandard tableau $T, \psi_{T}$ is a bijection.

Proof. Fix a tableau $T$ and denote its shape by $\mu$. Consider a reverse plane partition $R$ of shape $\lambda$ whose reading word is equivalent to that of $T$ and set $F=\psi_{T}(R)$. Let

$$
R=R_{0} \rightarrow \operatorname{co}(R)=R_{1} \rightarrow \operatorname{co}\left(R_{1}\right)=R_{2} \rightarrow \cdots \rightarrow R_{r}=T
$$

and note that this process does terminate in $T$ since each contraction preserves the equivalence class of the reading word. By construction, $F$ has weakly increasing rows, is of the proper shape, and has only entries less than $i$ in row $i$. To see that the entries of $F$ are strictly increasing up columns, it is enough to note that the reverse jeu-de-taquin paths of two cells from the same row cannot intersect. Hence $F$ is an elegant filling. To invert this procedure, we repeatedly perform dilation on $T$, by placing an empty marker in a cell of $\lambda / \mu$, and sliding this marker into $T$, terminating at the row indicated by the corresponding entry of $F$. The order these contractions are performed is determined first by the entries of $F$ (ordered smallest to largest) breaking ties by proceeding from left to right.

Corollary 5.10. Theorem 5.2 follows from Theorem 3.5.

Proof. We apply Theorem 3.5 to the trivial expansion (18) to find that

$$
s_{\mu}=\sum_{S \in \mathbb{S}}(-1)^{\varepsilon(S)} g_{s h(S)}=\sum_{R \in \mathbb{R}} G_{s h(R)},
$$

where $\mathbb{S}$ is the set of all set-valued tableaux whose reading word is Knuth equivalent to $w(T)$ and $\mathbb{R}$ is the set of all reverse plane partitions whose reading word is Knuth equivalent to $w(T)$. The results then follow from Propositions 5.6 and 5.9.

Corollary 5.11. Theorem 3.5 follows from Theorem 5.2.

Proof. Given Theorem 5.2, we reinterpret the coefficients using Propositions 5.6 and 5.9 to obtain (19). Our claim follows by linear extension.

\section{Applications}

Here we give a direct combinatorial characterization for the $G$ - and $g$-expansions of products of Schur functions and certain Macdonald polynomials. Theorem 3.5 can be applied to any function $f$ with a tableaux Schur expansion to obtain the $G$ - and $g$-expansion for $f$. The expansion coefficients are given by the enumeration of reverse plane partitions (resp. set-valued tableaux) whose reading word is Knuth equivalent to prescribed sets of tableaux. We use such descriptions as a springboard for finding combinatorial interpretations for the coefficients that avoid Knuth equivalence. 


\subsection{Littlewood-Richardson expansions}

Perhaps the most classical example of a tableaux Schur expansion is the LittlewoodRichardson rule for multiplying Schur functions. The coefficients in

$$
s_{\mu} s_{\nu}=\sum_{\lambda} c_{\mu \nu}^{\lambda} s_{\lambda}
$$

have beautiful combinatorial descriptions, count multiplicities of the irreducible $G L(n, \mathbb{C})$ module $V^{\lambda}$ of highest weight $\lambda$ in the tensor product of $V^{\mu} \otimes V^{\nu}$, and encode the number of points in the intersection of certain Schubert varieties of the Grassmannian.

To describe the numbers $c_{\mu \nu}^{\lambda}$ requires that a certain Yamanouchi condition be described on words. A word $w=w_{1}, \ldots, w_{j}$ satisfies the Yamanouchi condition with respect to the letters $a_{1}<a_{2}<\cdots<a_{\ell}$ when, in every rightmost segment of $w, w_{i} w_{i+1} \ldots w_{j}$, the number of $a_{r}$ 's is greater than or equal to the number of $a_{r+1}$ 's for all $1 \leqslant r<\ell$. For example, the following words are examples of Yamanouchi words with respect to the letters $\{3,4,5\}$ :

$$
54433 \quad 45343 \quad 15432326 .
$$

The numbers $c_{\mu \nu}^{\lambda}$ can now be described as the number of skew semistandard Young tableaux of shape $\lambda / \mu$ which have evaluation $\nu$ and whose reading word satisfies the Yamanouchi condition. This was first stated by Littlewood-Richardson [19] and first proved by Schützenberger [28] and Thomas [31, 32]. Note that Theorem 3.5 cannot be applied directly to (21), since $c_{\mu \nu}^{\lambda}$ counts tableaux of shape $\lambda / \mu$, not shape $\lambda$. However, in this case, this is easily remedied.

We begin by defining $\mathbb{T}(\mu, \nu)$ as the set of tableaux with evaluation $\left(\mu_{1}, \ldots, \mu_{\ell}\right.$, $\left.\nu_{1}, \ldots, \nu_{k}\right)$ whose reading words satisfy the Yamanouchi condition with respect to the letters $1, \ldots,|\mu|$ and (separately) with respect to the letters $|\mu|+1, \ldots,|\mu|+|\nu|$.

Lemma 6.1. We have the tableaux Schur expansion

$$
s_{\mu} s_{\nu}=\sum_{T \in \mathbb{T}(\mu, \nu)} s_{s h(T)} .
$$

Proof. It is not hard to see that any tableau whose reading word satisfies the Yamanouchi condition with respect to the letters $1, \ldots,|\mu|$ must contain as a subtableau the unique tableau of shape and evaluation $\mu$. Hence there is a bijection between the tableaux counted by $c_{\mu \nu}^{\lambda}$ and those tableaux in $\mathbb{T}(\mu, \nu)$ of shape $\lambda$, given by adding $|\mu|$ to every letter and filling in the inner shape $\mu$ with the unique tableau of shape and evaluation $\mu$.

We can apply Theorem 3.5 to this tableaux Schur expansion to get the $G$ and $g$ expansion of $s_{\mu} s_{\nu}$ as a sum over elements of $\mathbb{S}(\mu, \nu)$ and $\mathbb{R}(\mu, \nu)$. In fact, the elements of $\mathbb{S}(\mu, \nu)$ and $\mathbb{R}(\mu, \nu)$ can be more directly characterized in terms of the Yamanouchi condition. 
Lemma 6.2. Consider $\mathbb{T}(\mu, \nu)$ as defined above. A set-valued tableau $S \in \mathbb{S}(\mu, \nu)$ (resp. $R \in \mathbb{R}(\mu, \nu)$ ) if and only if $S$ (resp. $R$ ) has evaluation $\left(\mu_{1}, \ldots, \mu_{\ell}, \nu_{1}, \ldots, \nu_{k}\right)$ and its reading word satisfies the Yamanouchi condition with respect to the letters $1, \ldots,|\mu|$ and (separately) with respect to the letters $|\mu|+1, \ldots,|\mu|+|\nu|$.

Proof. By definition, the reading word of $S \in \mathbb{S}(\mu, \nu)$ (resp. $R \in \mathbb{R}(\mu, \nu)$ ) is Knuth equivalent to an element of $\mathbb{T}(\mu, \nu)$. The result then follows from the fundamental property that the Yamanouchi property is preserved under Knuth equivalence [7].

Corollary 6.3. For partitions $\mu=\left(\mu_{1}, \ldots, \mu_{\ell}\right)$ and $\nu=\left(\nu_{1}, \ldots, \nu_{k}\right)$,

$$
\begin{aligned}
s_{\mu} s_{\nu} & =\sum_{S \in S(\mu, \nu)}(-1)^{\varepsilon(S)} g_{s h(S)} \\
& =\sum_{R \in R(\mu, \nu)} G_{s h(R)} .
\end{aligned}
$$

where $S(\mu, \nu)$ (resp. $R(\mu, \nu)$ ) is the set of set-valued tableaux (reverse plane partitions) with evaluation $\left(\mu_{1}, \ldots, \mu_{\ell}, \nu_{1}, \ldots, \nu_{k}\right)$ whose reading words satisfy the Yamanouchi condition with respect to the letters $1, \ldots,|\mu|$ and (separately) with respect to the letters $|\mu|+1, \ldots,|\mu|+|\nu|$.

\subsection{Hall-Littlewood symmetric functions}

An acclaimed family of functions with a tableaux Schur expansion is the Hall-Littlewood basis $\left\{H_{\lambda}[X ; t]\right\}_{\lambda}$. These are a basis for $\Lambda$ over the polynomial ring $\mathbb{Z}[t]$ that reduces to the homogeneous basis when the parameter $t$ is set to 1 . These often are denoted by $\left\{Q_{\lambda}^{\prime}[X ; t]\right\}$ in the literature ([25]). Hall-Littlewood polynomials arise and can be defined in various contexts such as the Hall Algebra, the character theory of finite linear groups, projective and modular representations of symmetric groups, and algebraic geometry. We define them here via a tableaux Schur expansion due to Lascoux and Schützenberger [20].

The key notion is the charge statistic on semistandard tableaux. This is given by defining charge on words and then defining the charge of a tableau to be the charge of its reading word. For our purposes, it is sufficient to define charge only on words whose evaluation is a partition. We begin by defining the charge of a word with weight $(1,1, \ldots, 1)$, or a permutation. If $w$ is a permutation of length $n$, then the charge of $w$ is given by $\sum_{i=1}^{n} c_{i}(w)$ where $c_{1}(w)=0$ and $c_{i}(w)$ is defined recursively as

$$
c_{i}(w)=c_{i-1}(w)+\chi(i \text { appears to the right of } i-1 \text { in } w) .
$$

Here we have used the notation that when $P$ is a proposition, $\chi(P)$ is equal to 1 if $P$ is true and 0 if $P$ is false.

Example 6.4. $\operatorname{ch}(3,5,1,4,2)=0+1+1+2+2=6$. 
We will now describe the decomposition of a word with partition evaluation into charge subwords, each of which are permutations. The charge of a word will then be defined as the sum of the charge of its charge subwords. To find the first charge subword $w^{(1)}$ of a word $w$, we begin at the right of $w$ (i.e., at the last element of $w$ ) and move leftward through the word, marking the first 1 that we see. After marking a 1, we continue to travel to the left, now marking the first 2 that we see. If we reach the beginning of the word, we loop back to the end. We continue in this manner, marking successively larger elements, until we have marked the largest letter in $w$, at which point we stop. The subword of $w$ consisting of the marked elements (with relative order preserved) is the first charge subword. We then remove the marked elements from $w$ to obtain a word $w^{\prime}$. The process continues iteratively, with the second charge subword being the first charge subword of $w^{\prime}$, and so on.

Example 6.5. Given $w=(5,2,3,4,4,1,1,1,2,2,3)$, the first charge subword of $w$ are the bold elements in $(\mathbf{5}, \mathbf{2}, 3,4, \mathbf{4}, 1,1, \mathbf{1}, 2,2, \mathbf{3})$. If we remove the bold letters, the second charge subword is given by the bold elements in $(\mathbf{3}, \mathbf{4}, 1, \mathbf{1}, 2, \mathbf{2})$. It is now easy to see that the third and final charge subword is $(\mathbf{1}, \mathbf{2})$. Thus we have the following computation of the charge of $w$ :

$$
\begin{aligned}
\operatorname{ch}(w) & =\operatorname{ch}(5,2,4,1,3)+\operatorname{ch}(3,4,1,2)+\operatorname{ch}(1,2) \\
& =(0+0+1+1+1)+(0+1+1+2)+(0+1) \\
& =8
\end{aligned}
$$

Since $w$ is the reading word of the tableau

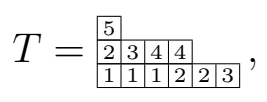

we find that the $\operatorname{ch}(T)=8$.

An important property of the charge function is that it is compatible with RSK:

Property 6.6. [20] For all words $w$, $\operatorname{ch}(w)=\operatorname{ch}(R S K(w))$.

Definition 6.7. The Hall-Littlewood polynomial $H_{\mu}[X ; t]$ is defined by

$$
H_{\mu}[X ; t]=\sum_{T \in \mathbb{T}(\mu)} t^{\operatorname{ch}(T)} s_{s h(T)}
$$

where $\mathbb{T}(\mu)$ is the set of all tableaux of evaluation $\mu$.

Note that when $t=1$, Definition 6.7 reduces to equation (6) implying that $H_{\mu}[X ; 1]=$ $h_{\mu}$.

In the same spirit that the charge of a semi-standard tableau is given by the charge of its reading word, we define the charge of a set-valued tableau and of a reverse plane partition by the taking the charge of their reading words. 
Example 6.8. Since the words of the set valued tableau

\begin{tabular}{|l|c|c|c|c|c|}
\hline 2 & 3 & 4,5 & & \\
\hline 1 & 1 & 1 & 2 & 2 & 3,4 \\
\hline
\end{tabular}

and the reverse plane partition

\begin{tabular}{|c|c|c|c|c|}
\hline 5 & & & & \\
\hline 2 & 3 & & & \\
\hline 1 & 3 & & 4 & \\
\hline 1 & 1 & & 2 & $\begin{array}{lll}2 & 3 \\
\end{array}$ \\
\hline
\end{tabular}

are both given by $w=(5,2,3,4,4,1,1,1,2,2,3)$, the charge of both of these objects is 8 .

Corollary 6.9. The Hall-Littlewood functions can be written in terms of G-functions as

$$
H_{\mu}[X ; t]=\sum_{\lambda} \sum_{R \in R P P(\lambda, \mu)} t^{\operatorname{ch}(R)} G_{\lambda},
$$

and in terms of $g$-functions as

$$
H_{\mu}[X ; t]=\sum_{\lambda}(-1)^{|\mu|-|\lambda|} \sum_{S \in S V T(\lambda, \mu)} t^{\operatorname{ch}(S)} g_{\lambda} .
$$

Proof. The tableaux Schur expansion (23) for Hall-Littlewood polynomials becomes

$$
\begin{aligned}
H_{\mu}[X ; t] & =\sum_{R \in \mathbb{R}(\mu)} t^{\operatorname{ch}(R S K(w(R))} G_{s h(R)} \\
& =\sum_{S \in \mathbb{S}(\mu)} t^{\operatorname{ch}(R S K(w(S))}(-1)^{\varepsilon(S)} g_{s h(S)}
\end{aligned}
$$

by Theorem 3.5. By Property 6.6, we have that $\operatorname{ch}(R S K(w))=\operatorname{ch}(w)$. Moreover, the set $\mathbb{R}(\mu)$ (resp. $\mathbb{S}(\mu))$ is none other than reverse plane partitions (resp. set-valued tableaux) of evaluation $\mu$.

\subsection{Macdonald polynomials}

The generalization of the Hall-Littlewood polynomials to a two parameter family of symmetric functions gives a hotly studied problem in the area of tableaux Schur expansions. Ideas impacting theories ranging from Hilbert schemes of points in the plane to quantum cohomology have come forth from the study of the Schur expansion coefficients $K_{\lambda \mu}(q, t)$ in Macdonald polynomials:

$$
H_{\mu}[X ; q, t]=\sum_{\lambda} K_{\lambda \mu}(q, t) s_{\lambda} .
$$

Major progress in the combinatorial study of these coefficients during the last decade establishes that

$$
H_{\mu}[X ; q, t]=\sum_{T \in \mathbb{T}\left(1^{n}\right)} w t_{\mu}(T) s_{s h(T)},
$$


where $\mathbb{T}\left(1^{n}\right)$ is the set of standard tableaux and $w t_{\mu}$ is an unknown statistic associating some $t$ and $q$ power to each standard tableaux $[9,1]$.

The ongoing search for $w t_{\mu}$ has led to many exciting theories and a natural solution has been found in special cases. When $q=0, H_{\mu}[X ; 0, t]$ are the Hall-Littlewood polynomials, whose expansion was just described in the previous section in terms of charge. In addition to the Hall-Littlewood case, there is a tableaux Schur expansion when $q=1$. Again, we not only apply our theorem to obtain the $G$ - and $g$-expansions, but we can go further to provide a direct combinatorial interpretation for the expansion coefficients.

The formula for expanding $H_{\lambda}[X ; 1, t]$ into Schur functions was given by Macdonald [25] in terms of a refined charge statistic. In particular, for any standard tableau $T$, he defines

$$
\operatorname{ch}_{\mu}(T)=\sum_{i \geqslant 1} \operatorname{ch}\left(\rho_{i} T\right),
$$

where $\rho_{i} T$ is the restriction of $T$ to the $i t h$ segment of $[1, n]$ of length $\mu_{i}^{\prime}$ (with each entry decremented by $\left.\sum_{j<i} \mu_{j}^{\prime}\right)$.

Example 6.10. Let $\mu=(3,3,2,1)$ and

$$
T=\begin{array}{|l|l|l|l|}
\hline 9 & \multicolumn{3}{|c}{} \\
\hline 4 & 5 & 6 & 8 \\
\hline 1 & 2 & 3 & 7 \\
\hline
\end{array}
$$

Note that $\mu^{\prime}=(4,3,2)$ and thus $\operatorname{ch}\left(\rho_{1} T\right)=\operatorname{ch}(4123)=5, \operatorname{ch}\left(\rho_{2} T\right)=\operatorname{ch}(567)=\operatorname{ch}(123)=$ $3, \operatorname{ch}\left(\rho_{3} T\right)=\operatorname{ch}(98)=\operatorname{ch}(21)=0$. Hence $\operatorname{ch}_{(3,3,2,1)}(T)=5+3+0=8$.

Macdonald then proves that

$$
H_{\mu}[X ; 1, t]=\sum_{\lambda} \sum_{T \in S S T\left(\lambda, 1^{n}\right)} t^{c h_{\mu}(T)} s_{\lambda} .
$$

Extending the definition of $c h_{\mu}$ to set-valued tableaux and reverse plane partitions in the same way (by restricting and decrementing the entries of the reading word), Theorem 3.5 gives the $G$ - and $g$-expansions of the $H_{\mu}[X ; 1, t]$.

Corollary 6.11. The Macdonald polynomials when $q=1$ satisfy the expansions

$$
\begin{aligned}
H_{\mu}[X ; 1, t] & =\sum_{\lambda}(-1)^{|\mu|-|\lambda|} \sum_{S \in S V T\left(\lambda, 1^{n}\right)} t^{c h_{\mu}(S)} g_{\lambda} \\
& =\sum_{\lambda} \sum_{R \in R P P\left(\lambda, 1^{n}\right)} t^{c h_{\mu}(R)} G_{\lambda} .
\end{aligned}
$$

\section{Other applications and future work}

Here we give the $G$ - and $g$-expansion for $k$-atoms and Stanley symmetric functions where the expansion coefficients count reverse plane partitions (resp. set-valued tableaux) whose reading word is Knuth equivalent to prescribed sets of tableaux. We leave as open problems the task of describing the coefficients without using Knuth equivalence. 


\section{$7.1 \quad \mathrm{k}$-Atoms}

One study of the tableaux Schur expansion of Macdonald polynomials led to the discovery of a family of polynomials called atoms. To be precise, the [15] study restricted attention to the subspaces of Macdonald polynomials $\Lambda_{t}^{k}=\operatorname{span}\left\{H_{\lambda}[X ; q, t]\right\}_{\lambda_{1} \leqslant k}$, for fixed integer $k>0$. There, for each partition with no part larger than $k$, a $k$-atom was introduced and defined as

$$
s_{\mu}^{(k)}[X ; t]=\sum_{T \in \mathcal{A}_{\mu}^{k}} t^{c h(T)} s_{s h(T)},
$$

for certain prescribed sets of tableaux $\mathcal{A}_{\mu}^{k}$ (see [15]). It was conjectured that any Macdonald polynomial in $\Lambda_{t}^{k}$ can be decomposed as:

$$
H_{\lambda}[X ; q, t]=\sum_{\mu \in \mathcal{P}^{k}} K_{\mu \lambda}^{(k)}(q, t) s_{\mu}^{(k)}[X ; t] \quad \text { where } \quad K_{\mu \lambda}^{(k)}(q, t) \in \mathbb{N}[q, t]
$$

Moreover, it was conjectured that the element $s_{\mu}^{(k)}[X ; t]$ reduces simply to $s_{\mu}$ for $k \geqslant|\lambda|$, thus refining (28) since the expansion coefficients in (33) are $K_{\lambda \mu}(q, t)$ for large $k$.

The $k$-atoms are a perfect candidate for Theorem 3.5 since they have a tableaux Schur expansion by definition.

Corollary 7.1. For $\mathbb{T}(\mu, k)=\mathcal{A}_{\mu}^{k}$, we have that

$$
\begin{aligned}
s_{\mu}^{(k)}[X ; t] & =\sum_{S \in \mathbb{S}(\mu, k)}(-1)^{\varepsilon(S)} t^{\operatorname{ch}(S)} g_{s h(S)} \\
& =\sum_{R \in \mathbb{R}(\mu, k)} t^{\operatorname{ch}(R)} G_{s h(R)} .
\end{aligned}
$$

\subsection{Stanley symmetric functions}

Let $s_{1}, \ldots, s_{n-1}$ be the simple transpositions which generate the symmetric group $S_{n}$. To each reduced decomposition $\sigma=s_{i_{1}} \ldots s_{i_{n}}$, we associate the word $\left(i_{1}, \ldots, i_{n}\right)$. For $\sigma \in S_{n}$, let $R(\sigma)$ denote the set of words for reduced decompositions of $\sigma$. Stanley [29] defined symmetric functions

$$
F_{\sigma}=\sum_{w_{1} w_{2} \cdots w_{k} \in R(\sigma)} \sum_{\substack{i_{1} \leqslant i_{2} \leqslant \cdots \leqslant i_{k} \\ w_{j}>w_{j+1} \Longrightarrow i_{j}<i_{j+1}}} x_{i_{1}} \ldots x_{i_{k}} .
$$

Stanley showed that $F_{\sigma}$ is always a symmetric function, and conjectured that it is always Schur-positive. This conjecture was later proven by Edelman-Greene [5], and independently by Lascoux-Schützenberger [22]. Edelman-Greene gave a tableaux Schur expansion for the Stanley symmetric functions. 
Theorem $7.2([5])$.

$$
F_{\sigma}=\sum_{T \in \mathbb{T}(\sigma)} s_{s h(T)}
$$

over the set $\mathbb{T}(\sigma)$ of all tableaux whose reading word is a reduced word for $\sigma^{-1}$.

Since we have a tableaux Schur expansion, we can apply Theorem 3.5.

\section{Corollary 7.3.}

$$
F_{\sigma}=\sum_{S \in \mathbb{S}(\sigma)}(-1)^{\varepsilon(S)} g_{s h(S)},
$$

where $\mathbb{S}(\sigma)$ is the set of all set-valued tableaux with a reading word which is Knuth equivalent to a reduced word for $\sigma^{-1}$, and

$$
F_{\sigma}=\sum_{R \in \mathbb{R}(\sigma)} G_{s h(R)}
$$

where $\mathbb{R}(\sigma)$ is the set of all reverse plane partitions with a reading word which is Knuth equivalent to a reduced word for $\sigma^{-1}$.

It is worth noting that we cannot describe $\mathbb{S}(\sigma)$ as the set of all set-valued tableaux whose reading word is a reduced word for $\sigma^{-1}$. For example, consider

$$
S=\begin{array}{|c|c|}
\hline 3 \\
\hline 1,22,3
\end{array} .
$$

The reading word $w(S)=33212$ is clearly not the reduced word of any permutation, but $S$ is in $\mathbb{S}\left(s_{2} s_{1} s_{3} s_{2} s_{3}\right)$, since $w(R S K(w(S)))=32312$. On the other hand

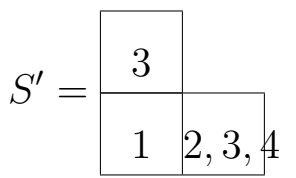

has reading word 34312 which is a reduced word for the inverse of the permutation $\left(s_{2} s_{1} s_{3} s_{4} s_{3}\right)$. However, the reading word of $R S K\left(w\left(S^{\prime}\right)\right)$ is 43312 , so $S^{\prime}$ is not in $\mathbb{S}(\sigma)$ for any $\sigma$.

A similar phenomenon occurs with reverse plane partitions. For example, with

$$
T=\begin{array}{|l|l|}
\hline 2 & 2 \\
\hline 1 & 2 \\
\hline 1 & 3 \\
\hline
\end{array}
$$

$w(T)=2213$ which is not a reduced word. However, applying RSK gives a tableau whose reading word is the reduced word 2123. On the other hand, given instead

$$
T^{\prime}=\begin{array}{|l|l|}
\hline 3 & 4 \\
\hline 1 & 3 \\
\hline 1 & 2 \\
\hline
\end{array},
$$

THE ELECTRONic Journal of COMBinatorics 19(4) (2012), \#P39 
we have that $w\left(T^{\prime}\right)=34312$ is reduced, but the insertion gives a tableau whose reading word is 43312 .

Although Corollary 7.3 gives an interpretation for the $g$ - and $G$-expansions of a Stanley symmetric function, a characterization of the set-valued tableaux and reverse plane partitions appearing in these expansions that does not involve RSK-insertion remains an interesting open problem.

\section{Identities}

Proposition 8.1. Given any function $f_{\alpha}$ where

$$
f_{\alpha}=\sum_{T \in \mathbb{T}(\alpha)} w t(T) s_{s h(T)}
$$

we have that

$$
\sum_{S \in \mathbb{S}(\alpha)}(-1)^{\varepsilon(S)} w t(S)=w t\left(S_{0}\right)
$$

where $S_{0}$ is the tableau of row shape.

Proof. We do this by constructing a sign-reversing, weight-preserving involution on $\mathbb{S}(\alpha)$ where $S_{0}$ is the only fixed point. The appropriate candidate is defined as follows; let $n$ denote the largest letter in $S$ and set $x=n$. Let $\operatorname{row}(S)$ be the highest row of $S$ containing $x$ and determine which of the following cases occurs:

1. there is an $x$ in a multi-element cell of row $\operatorname{row}(S)$

2. $\operatorname{row}(S) \neq 1$ and no multi-element cells in $\operatorname{row} \operatorname{row}(S)$ or $\operatorname{row}(S)-1$ contain $x$

3. $\operatorname{row}(S)=1$ and there is no $x$ in a multi-element cell of row 1 .

When $S$ satisfies condition (1), construct $S^{\prime}$ from $S$ by moving the $x$ in this multi-element cell to the end of $\operatorname{row}(S)+1$. When we have condition (2), construct $S^{\prime}$ from $S$ by moving the $x$ from the end of row $\operatorname{row}(S)$ into the rightmost cell of $\operatorname{row}(S)-1$ containing only letters strictly smaller than $x$. In the last case (3), we replace $x$ by $x-1$ and determine which case is satisfied by $S$, starting from the point that we let $\operatorname{row}(S)$ denote the highest row containing $x$. The fixed points are semi-standard tableaux of row shape (those with all letters in row 1 and no multi-element cells).

Corollary 8.2. The sum of the expansion coefficients $d_{\mu \lambda}(t)$ in

$$
H_{\mu}[X ; t]=\sum_{\lambda} d_{\mu \lambda}(t) g_{\lambda}
$$

is $t^{n(\mu)}$.

Proof. There is only one semi-standard row shape tableau $T$ of weight $\mu$ and charge $(T)=$ $n(\mu)$. 


\section{Related work}

In [12], Lascoux studies transformations on symmetric functions under the shift of power sums $p_{i} \rightarrow p_{i} \pm 1$. The application of such a transformation to the Hall-Littlewood polynomials leads to an expansion for transformed Hall-Littlewood polynomials in terms of plane partitions. Finding the precise connection between this expansion and the expansion of a Hall-Littlewood polynomial $H_{\mu}[X ; t]$ given in Corollary 6.9 is an interesting problem.

The Schubert representatives for the K-theory of affine Grassmannians and their dual in the nil Hecke ring are given by a refinement of the $G$ and the $g$-functions to families, $G^{k}$ and $g^{k}[24,26]$. These too are inhomogenous functions, but they are indexed by an extra integer parameter $k>0$ and now, the dual $k$-Schur functions [17] and $k$-Schur functions [16] are the lowest and highest degree terms, respectively. There is a tableaux definition for $G^{k}$ in the spirit of Definition 2.7 for Schur functions [26] giving rise to many problems regarding the tableaux combinatorics of the $G^{k} / g^{k}$ families. One direction along these lines would be to find the $G^{k} / g^{k}$-expansions of functions with a tableaux- $k$-Schur or tableaux-dual- $k$-Schur expansion.

\section{References}

[1] Sami H Assaf. Dual equivalence graphs I: A combinatorial proof of LLT and Macdonald positivity. arXiv:1005.3759v1, 2007.

[2] Francois Bergeron. Algebraic Combinatorics and Coinvariant Spaces. A K Peters/CRC Press, 2009.

[3] Anders Skovsted Buch. A Littlewood-Richardson rule for the $K$-theory of Grassmannians. Acta Math., 189(1):37-78, 2002.

[4] G. de B Robinson. On the representations of $S_{n}$. American Journal of Mathematics, 60:745-760, 1938.

[5] Paul Edelman and Curtis Greene. Balanced tableaux. Advances in Mathematics, 63(1):42-99, 1987.

[6] Sergey Fomin and Anatol N. Kirillov. Grothendieck polynomials and the Yang-Baxter equation. In Formal power series and algebraic combinatorics/Séries formelles et combinatoire algébrique, pages 183-189. DIMACS, Piscataway, NJ, 1994.

[7] William Fulton. Young tableaux. With applications to representation theory and geometry. Cambridge University Press, Cambridge, United Kingdom, 1991.

[8] A. M. Garsia and C. Procesi. On certain graded $S_{n}$-modules and the $q$-Kostka polynomials. Adv. Math, 94:82-138, 1992.

[9] Mark Haiman. Hilbert schemes, polygraphs, and the Macdonald positivity conjecture. Journal of the American Mathematics Society, 14:941-1006, 2001.

[10] Philip Hall. The algebra of partitions. Proc. of the 4 th Canadian Math. Congress, pages $147-159,1959$. 
[11] Donald E Knuth. Permutations, matrices, and generalized young tableaux. Pacific Journal of Mathematics, 34:709-727, 1970.

[12] Alain Lascoux. Adding \pm 1 to the argument of a Hall-Littlewood polynomial, volume B54n. Séminaire Lotharingien de Combinatoire, 2007.

[13] C. Lenart. Combinatorial aspects of the $k$-theory of Grassmannians. Ann Combin, 4:67-82, 2000.

[14] D. E. Littlewood. On certain symmetric functions. Proc. London Math. Soc., 11:485498, 1961.

[15] L. Lapointe, A. Lascoux, and J. Morse. Tableau atoms and a new Macdonald positivity conjecture. Duke Math J, 116(1):103-146, 2003.

[16] L. Lapointe and J. Morse. A $k$-tableaux characterization for $k$-Schur functions. Adv Math, pages 183-204, 2007.

[17] L. Lapointe and J. Morse. Quantum cohomology and the $k$-Schur basis. Trans Amer Math Soc, 360:2021-2040, 2008.

[18] Thomas Lam and Pavlo Pylyavskyy. Combinatorial Hopf algebras and $K$-homology of Grassmannians. Int. Math. Res. Not. IMRN, (24):Art. ID rnm125, 48, 2007.

[19] D.E. Littlewood and A.R. Richardson. Group characters and algebra. Phil. Trans. Royal Soc. A (London), 233:99-141, 1934.

[20] Alain Lascoux and Marcel-Paul Schützenberger. Sur une conjecture de H. O. Foulkes. C. R. Acad. Sci. Paris Sér. A-B, 286(7):A323-A324, 1978.

[21] A. Lascoux and M.-P. Schützenberger. Le monoide plaxique. Noncommutative structures in algebra and geometric combinatorics, 109:129-156, 1981.

[22] Alain Lascoux and Marcel-Paul Schützenberger. Structure de Hopf de l'anneau de cohomologie et de l'anneau de Grothendieck d'une variété de drapeaux. C. R. Acad. Sci. Paris Sér. I Math., 295(11):629-633, 1982.

[23] Alain Lascoux and Marcel-Paul Schützenberger. Symmetry and flag manifolds. In Invariant theory (Montecatini, 1982), volume 996 of Lecture Notes in Math., pages 118-144. Springer, Berlin, 1983.

[24] T. Lam, A. Schilling, and M. Shimozono. K-theory Schubert calculus of the affine Grassmannian. Compositio Math, 146:811-852, 2010.

[25] Ian G. Macdonald. Symmetric Functions and Hall Polynomials. The Clarendon Press, Oxford University Press, New York, second edition, 1995. With contributions by A. Zelevinsky.

[26] Jennifer Morse. Combinatorics of the $K$-theory of affine Grassmannians. Adv Math, 2009.

[27] C. Schensted. Longest increasing and decreasing subsequences. Canadian Journal of Mathematics. Journal Canadien de Mathématiques, 13:179-191, 1961.

[28] M.-P. Schützenberger. La correspondance de Robinson. In Combinatoire et représentation du groupe symétrique (Actes Table Ronde CNRS, Univ. Louis-Pasteur 
Strasbourg, Strasbourg, 1976), pages 59-113. Lecture Notes in Math., Vol. 579. Springer, Berlin, 1977.

[29] Richard P Stanley. On the number of reduced decompositions of elements of Coxeter groups. European Journal of Combinatorics, 5(4):359-372, 1984.

[30] Richard P. Stanley. Enumerative Combinatorics, volume 2. Cambridge University Press, Cambridge, United Kingdom, 1999.

[31] Glânffrwd P. Thomas. Baxter algebras and Schur functions. PhD thesis, Unversity College of Swansea, 1974.

[32] Glânffrwd P. Thomas. On a construction of Schützenberger. Discrete Math., 17(1):107-118, 1977. 